\title{
Rural and remote health: a progress report
}

\section{Doing better, but we still need to sort out who is responsible for what and strengthen primary care}

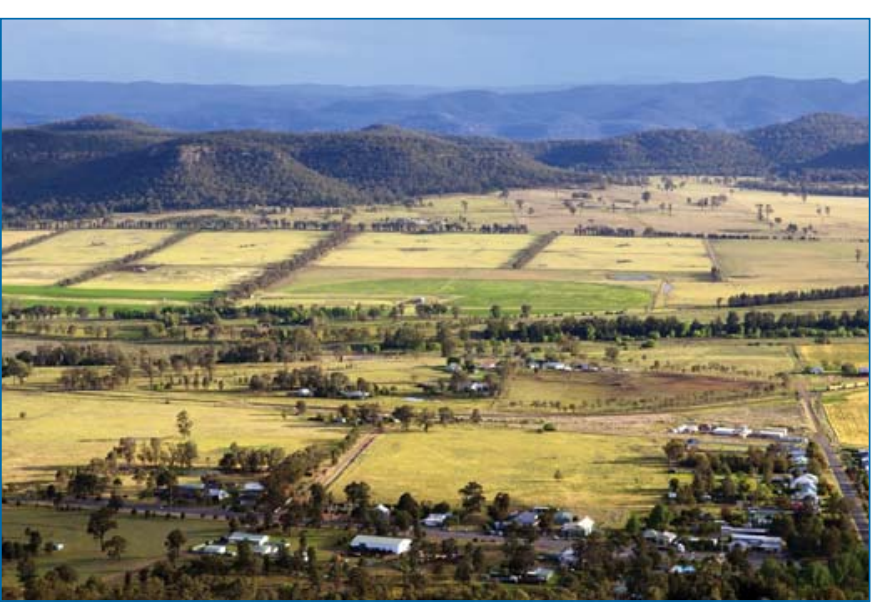

ollowing an active period of policy development and implementation in the 1990s, ${ }^{1}$ there have been two national great leaps forward that tackled geographical health inequalities in Australia in the new century. In 2000, in the context of growing disaffection in the rural electorate, ${ }^{2}$ the then health minister, Michael Wooldridge, was responsible for a federal Budget centrepiece of \$562 million, the Regional Health Strategy: More Doctors, Better Services. The package included the establishment of university departments of rural health and rural clinical schools, as well as retention incentives for rural doctors. The second leap resulted from the balance of power held by the two rural independents in 2010. Their agreement with the minority Labor government for regional development included investment of some $\$ 2.33$ billion in rural and remote health infrastructure, including regional cancer centres. As ever, the political imperative was key.

\section{Where are we now?}

As the fiscal belt tightens and we scan for a similar policy window of opportunity for rural health, it is timely to reflect on progress.

There is no doubt that there have been some very significant gains since 2000. We have moved beyond a deficit view of rural health and there is a stronger recognition that our tough context provides an "incubator for developing and testing new models of care and expanded scopes of practice". ${ }^{3}$ Persistent advocacy from groups like the National Rural Health Alliance (NRHA) has resulted in increased public knowledge about the health inequalities between rural and metropolitan Australia. This health inequality has a complex aetiology that includes social determinants, disease risk factors and a proportionally larger Indigenous population. Nevertheless,
John Wakerman MTH, FAFPHM, FACRRM

Flinders University, Darwin, NT.

john.wakerman@ flinders.edu.au

doi: 10.5694/mjal5.00398 
access to health services is still critically important. There are examples of exemplary services that enhance access to primary health care. ${ }^{4}$ Some of the best examples of services that result in improved outcomes support the most remote Indigenous outstations. ${ }^{5}$

As highlighted above, rural and remote health infrastructure has improved access to primary and acute care, and there is emerging evidence of its impact. ${ }^{6}$

While medical workforce maldistribution and problems with training continuity (from student to prevocational to specialist training) persist, there are some promising trends resulting from the current suite of policies, including increasing numbers of medical students of rural origin and lengthening time of training in rural areas. ${ }^{7}$ This is a salutary example of the importance of long-term investment in infrastructure and workforce development. Proposed changes in the more equitable distribution of general practitioner incentives as the result of changes in the rural classification system to the Modified Monash Model should provide another medical workforce boost to small rural and remote communities. ${ }^{8}$

\section{"Many of our policy settings are right, and we need to hold the course for the long term"}

Despite these significant investments, the gap in health funding for rural and remote areas persists. ${ }^{9}$ The NRHA estimates a primary and aged care service deficit in the order of $\$ 3$ billion. ${ }^{10}$ Less access to primary health care is reflected in lower Medicare expenditure, higher compensatory hospitalisation and more potentially preventable hospital admissions with increasing remoteness. ${ }^{9}$ Although activity-based hospital funding is adjusted for rurality and Indigenous population, there is no adequate formula to adjust primary health care funding appropriately for morbidity and the higher costs of providing services to a highly dispersed population. The Mason health workforce review saliently comments on the need to refocus on prevention and "to move beyond a focus on specialist medicine and acute care beds, to appropriate generalist skills, team based community care and the training and development of the nursing and allied health workforce" ${ }^{\prime}$.

Integration and optimal coordination of services are still hampered by divided federal and state responsibilities. There was a missed opportunity to "end the blame game" with national health care reform initiated by the Rudd government. At the same time, the health care reforms have resulted in increased community participation through local hospital networks, and some regionalised health service models that allow for a degree of improved coordination.

\section{Where to next?}

Many of our policy settings are right, and we need to hold the course for the long term. Australia has an effective rural and remote academic infrastructure that is the envy of the world. Service infrastructure, including telehealth capacity, continues to improve. The main outstanding challenges relate to macropolicy — who is responsible for what - and its impact on coordination and adequate funding of services, especially primary health care services.

The available evidence from Australia and abroad is that cost savings result from increasing access to primary care and thereby decreasing potentially preventable hospitalisation. ${ }^{4,11,12}$ So we need to strengthen primary care, avoid introducing barriers to it, and strengthen preventive care across remote and rural Australia through appropriate investment to create effective, integrated regional models of care that are fit for context. We know how to do this, but need to get better at generalising what we know works well.

Acknowledgements: I am a Chief Investigator of the Centre of Research Excellence (CRE) in Rural and Remote Primary Health Care. The CRE is funded by the Australian Primary Health Care Research Institute (APHCRI), which is supported by a grant from the Australian Government Department of Health. The information and opinions in this article do not necessarily reflect the views or policy of APHCRI or the Australian Government Department of Health.

Competing interests: My institution receives funding from the Australian Department of Health Rural Health Multidisciplinary Training Program. I am a member of the Health and Hospitals Fund Advisory Board.

Provenance: Not commissioned; externally peer reviewed.

References are available online at www.mja.com.au. 
1 Humphreys JS, Gregory G. Celebrating another decade of progress in rural health: what is the current state of play? Aust J Rural Health 2012; 20: 156-163.

2 Tonts M, McKenzie FH. Neoliberalism and changing regional policy in Australia. Int Planning Stud 2005; 10: 183-200.

3 Australia's Health Workforce. Productivity Commission research report. Canberra: Productivity Commission, 2005: 204. http://www.pc.gov.au/inquiries/completed/healthworkforce/report/healthworkforce.pdf (accessed Apr 2015).

4 Wakerman J, Humphreys JS, Wells R, et al. Features of effective primary health care models in rural and remote Australia: a case-study analysis. Med J Aust 2009; 191: 88-91.

5 Rowley KG, O'Dea K, Anderson I, et al. Lower than expected morbidity and mortality for an Australian Aboriginal population: 10-year follow-up in a decentralised community. Med J Aust 2008; 188: 283-287.

6 PricewaterhouseCoopers. Evaluating the Health and Hospitals Fund. Detailed evaluation findings. Canberra: Australian Government Department of Health, 2014. http://www.health. gov.au/internet/main/publishing.nsf/Content/DC7F68B2307 16571CA257BF0001DC49E/\$File/HHF\%20-\%20Detailed\%20 Evaluation\%20Findings.pdf (accessed Apr 2015).

7 Kondalsamy-Chennakesavan S, Eley DS, Ranmuthugala G, et al. Determinants of rural practice: positive interaction between rural background and rural undergraduate training. Med J Aust 2015; 201: 41-45.

8 Mason J. Review of Australian Government Health Workforce Programs. Canberra: Australian Government Department of Health, 2013.

9 Australian Institute of Health and Welfare. Australian health expenditure by remoteness: a comparison of remote, regional and city health expenditure. Health and welfare expenditure series no. 50. Canberra: AlHW, 2010. (AlHW Cat. No. HWE 50.)

10 National Rural Health Alliance. Australia's health system needs re-balancing: a report on the shortage of primary care services in rural and remote areas. Canberra: NRHA, 2011. http:// ruralhealth.org.au/sites/default/files/documents/nrha-policydocument/position/pos-full-complementary-report-27-feb-11. pdf (accessed Mar.2015).

1 Zhao Y, Thomas SL, Guthridge SL, Wakerman J. Better health outcomes at lower costs: the benefits of primary care utilisation for chronic disease management in remote Indigenous communities in Australia's Northern Territory. BMC Health Serv Res 2014; 14: 463.

12 Rosano A, Loha CA, Falvo R, et al. The relationship between avoidable hospitalization and accessibility to primary care: a systematic review. Eur J Public Health 2013; 23: 356-360. 